University of Nebraska - Lincoln

DigitalCommons@University of Nebraska - Lincoln

$4-1-2012$

\title{
Tailoring the immune response by targeting C-type lectin receptors on alveolar macrophages using "pathogen-like" amphiphilic polyanhydride nanoparticles
}

\author{
Ana V. Chavez-Santicoy \\ lowa State University, apchavez@iastate.edu \\ Rajarshi Roychoudhury \\ lowa State University \\ Nicola L.B. Pohl \\ lowa State University, npohl@iastate.edu \\ Michael J. Wannemeuhler \\ lowa State University, mjwannem@iastate.edu \\ Balaji Narasimhan \\ lowa State University, nbalaji@iastate.edu \\ See next page for additional authors \\ Follow this and additional works at: https://digitalcommons.unl.edu/gfiraimertait \\ Part of the Medical Sciences Commons
}

Chavez-Santicoy, Ana V.; Roychoudhury, Rajarshi; Pohl, Nicola L.B.; Wannemeuhler, Michael J.; Narasimhan, Balaji; and Ramer-Tait, Amanda E., "Tailoring the immune response by targeting C-type lectin receptors on alveolar macrophages using "pathogen-like" amphiphilic polyanhydride nanoparticles"

(2012). Dr. Amanda Ramer-Tait Publications. 2.

https://digitalcommons.unl.edu/gfiraimertait/2

This Article is brought to you for free and open access by the Gut Function Initiative at DigitalCommons@University of Nebraska - Lincoln. It has been accepted for inclusion in Dr. Amanda Ramer-Tait Publications by an authorized administrator of DigitalCommons@University of Nebraska - Lincoln. 


\section{Authors}

Ana V. Chavez-Santicoy, Rajarshi Roychoudhury, Nicola L.B. Pohl, Michael J. Wannemeuhler, Balaji Narasimhan, and Amanda E. Ramer-Tait 


\title{
Tailoring the immune response by targeting C-type lectin receptors on alveolar macrophages using "pathogen-like" amphiphilic polyanhydride nanoparticles
}

\author{
Ana V. Chavez-Santoscoy, ${ }^{1}$ Rajarshi Roychoudhury, ${ }^{2}$ Nicola L.B. Pohl, ${ }^{1,2}$ \\ Michael J. Wannemuehler, ${ }^{3}$ Balaji Narasimhan, ${ }^{1}$ and Amanda E. Ramer-Tait ${ }^{3}$ \\ 1. Department of Chemical and Biological Engineering, Iowa State University, Ames, IA 50011, USA \\ 2. Department of Chemistry, Iowa State University, Ames, IA 50011, USA \\ 3. Department of Veterinary Microbiology and Preventive Medicine, Iowa State University, Ames, IA 50011, USA \\ Corresponding author — Balaji Narasimhan, email nbalaji@iastate.edu
}

\begin{abstract}
C-type lectin receptors (CLRs) offer unique advantages for tailoring immune responses. Engagement of CLRs regulates antigen presenting cell (APC) activation and promotes delivery of antigens to specific intracellular compartments inside APCs for efficient processing and presentation. In these studies, we have designed an approach for targeted antigen delivery by decorating the surface of polyanhydride nanoparticles with specific carbohydrates to provide pathogen-like properties. Two conserved carbohydrate structures often found on the surface of respiratory pathogens, galactose and di-mannose, were used to functionalize the surface of polyanhydride nanoparticles and target CLRs on alveolar macrophages (AM $\varphi$ ), a principle respiratory tract APC. Co-culture of functionalized nanoparticles with $\mathrm{AM} \varphi$ significantly increased cell surface expression of MHC I and II, CD86, CD40 and the CLR CIRE over non-functionalized nanoparticles. Di-mannose and galactose functionalization also enhanced the expression of the macrophage mannose receptor (MMR) and the macrophage galactose lectin, respectively. This enhanced AM $\varphi$ activation phenotype was found to be dependent upon nanoparticle internalization. Functionalization also promoted increased AM $\varphi$ production of the pro-inflammatory cytokines IL-1 $\beta$, IL- 6 and TNF- $\alpha$. Additional studies demonstrated the requirement of the MMR for the enhanced cellular uptake and activation provided by the di-mannose functionalized nanoparticles. Together, these data indicate that targeted engagement of MMR and other CLRs is a viable strategy for enhancing the intrinsic adjuvant properties of nanovaccine adjuvants and promoting robust pulmonary immunity.
\end{abstract}

Keywords: Polyanhydrides, Nanoparticles, Carbohydrates, Alveolar macrophages

\section{Introduction}

Acute respiratory infections cause 4.25 million deaths worldwide every year. ${ }^{1}$ A critical need exists for the development of efficacious intranasal vaccines against respiratory pathogens capable of inducing robust and protective mucosal immunity. In this regard, there is growing interest in the development of vaccines that can be easily administered to the site of infection in order to elicit both local and systemic immune responses. ${ }^{2-5}$

The study of alveolar macrophages $(\mathrm{AM} \varphi)$, a type of antigen presenting cell (APC) in the respiratory tract, is central to the development of intranasal vaccines. AM $\varphi$ constitute more than $80 \%$ of the total cells obtained by bronchoalveolar lavage of a healthy individual and they constitutively migrate from the lung to the draining lymph nodes (DLN) ${ }^{6-8}$ Indeed, $\mathrm{AM} \varphi$ containing bacteria appear in the pulmonary DLN prior to the onset of pathogen- induced DC migration, thereby making them integral to the establishment of protective pulmonary immune responses. ${ }^{6} \mathrm{AM} \varphi$ are equipped to detect pathogens with the aid of pattern recognition receptors (PRRs) that recognize pathogen-associated molecular patterns (PAMPs). ${ }^{9}$ One family of PRRs found on AM $\varphi$, known as Ctype lectin receptors (CLRs), recognize conserved carbohydrate structures, including mannose and galactose, found on the surface of many respiratory pathogens, such as Yersinia pestis, Mycobacterium tuberculosis, Streptococcus pneumoniae and influenza viruses. ${ }^{10-14}$ CLRs also function as phagocytic receptors and include members of the mannose receptor family and DC-SIGN (dendritic cell-specific intercellular adhesion molecule-3-grabbing non-integrin). ${ }^{15}$. Depending on the specific CLR, ligand binding initiates downstream signaling cascades that promote immune cell migration to the DLN as well as antigen processing and presentation via MHC I and/or MHC II to prime naïve T cells. ${ }^{16-20}$ 
Several research groups have explored CLR targeting as a vaccine design strategy to promote efficient delivery of cargo to intracellular compartments responsible for antigen processing and presentation. ${ }^{21-29}$ Many studies have demonstrated the effectiveness of using antibodies $^{22}$ or mannoproteins from pathogens ${ }^{26}$ to target CLRs and activate APCs. However, only a limited number report the use of carbohydrate-functionalized vaccine carriers as part of an improved adjuvant for intranasal vaccines. ${ }^{21,}{ }^{24}$ Work published by Jiang et al. indicated that alveolar macrophages could recognize mannosylated chitosan microparticles when delivered intranasally. ${ }^{24}$ Unfortunately, mechanistic studies demonstrating the engagement of the mannose receptor on $\mathrm{AM} \varphi$ by these particles were not performed. Here, we describe functionalization of polyanhydride nanoparticles with two conserved carbohydrate structures commonly found on the surface of respiratory pathogens, di-mannose and galactose. We also investigate the mechanisms by which these functionalized polyanhydride nanoparticles are internalized by and influence the activation of AM $\varphi$.

\section{Materials and methods}

\subsection{Materials}

The chemicals needed for monomer synthesis, polymerization and nanoparticle fabrication included 1,6-dibromohexane, triethylene glycol, 4-p-hydroxybenzoic acid, and 1-methyl-2-pyrrolidinone; these were purchased from Sigma-Aldrich (St. Louis, MO); 4-p-fluorobenzonitrile was obtained from Apollo Scientific (Cheshire, UK); toluene, sulfuric acid, acetonitrile, dimethyl formamide, acetic anhydride, methylene chloride, pentane, and potassium carbonate were obtained from Fisher Scientific (Fairlawn, NJ); $p$-carboxy benzoic acid (99+\%), and 1-methyl-2-pyrrolidinone, anhydrous (99+\%) were purchased from Aldrich (Milwaukee, WI). For ${ }^{1} \mathrm{H}$ NMR characterization, deuterated chemicals, including chloroform and dimethyl sulfoxide, were purchased from Cambridge Isotope Laboratories (Andover, MA).

\subsection{Monomer and polymer synthesis}

The 1,6-bis( $p$-carboxyphenoxy)hexane $\quad(\mathrm{CPH}) \quad$ and $\quad 1,8$-bis(pcarboxyphenoxy)-3,6-dioxaoctane (CPTEG) monomers were synthesized as previously described. ${ }^{30,31}$ Random copolymers with a 50:50 ratio of $\mathrm{CPH}$ and CPTEG were synthesized by melt polycondensation using an automated robotic deposition apparatus operated with LabVIEW ${ }^{\circledR}$ (National Instruments) as previously described. ${ }^{31-35}$ The chemical structure of the polymers was characterized via ${ }^{1} \mathrm{H}$ NMR with a Varian VXR $300 \mathrm{MHz}$ spectrometer (Varian Inc., Palo Alto, CA). Deuterated chloroform was used to dissolve the polymer and spectra were calibrated with respect to the chloroform peak $(\delta=7.26 \mathrm{ppm})$. The polymer molecular mass was determined using gel permeation chromatography (GPC). Samples were dissolved in HPLC-grade chloroform and separated on a Waters GPC chromatograph (Milford, MA) containing PL gel columns (Polymer Laboratories, Amherst, MA) comparing elution times to monodisperse polystyrene standards (Fluka, Milwaukee, WI).

\subsection{High throughput synthesis of carbohydrates}

A robotic set up was used for the iterative synthesis of linear $\alpha-1,2$-linked di-mannose with a fluorous allyl group using fluorous solid phase extraction (FSPE) serving as a model to obtain the di-mannoside. ${ }^{21,36-38}$ Carboxymethyl - di-mannose synthesis was performed by ozonolysis of the alkene ${ }^{39}$ followed by further oxidation with Jones reagent. ${ }^{37}$ Global deprotection under Birch reduction conditions ${ }^{40}$ produced the fully deprotected $\alpha-1,2$-linked di-mannose. In addition, $\beta-1-\mathrm{O}$-allylated galactose was prepared from $\beta$-penta-O-acetylated galactose using allyl alcohol and $\mathrm{BF}_{3}$. $\mathrm{OEt}_{2}$. Eight equivalents of $\mathrm{NaIO}_{4}$ utilized under ruthenium-catalyzed Sharpless conditions $s^{41,42}$ produced the desired acid in $91 \%$ yield. Subsequent deacetylation under mild conditions using $\mathrm{K}_{2} \mathrm{CO}_{3}$ provided the desired fully deprotected galactoside.

\subsection{High-throughput synthesis and characterization of functionalized nanoparticles}

The fabrication of functionalized 50:50 CPTEG:CPH nanoparticles was performed via an anti-solvent nanoencapsulation method using an automated robotic deposition apparatus operated by LabVIEW ${ }^{\circledR}$. 32, 33, 43 Galactose and dimannose residues were conjugated to the surface of nanoparticles by a modified and optimized two-step amine carboxylic acid coupling reaction. ${ }^{21}$ Briefly, the first reaction was performed at $4{ }^{\circ} \mathrm{C}$ by incubating the nanoparticle suspension $(100 \mathrm{mg} / \mathrm{mL})$ with 12 equivalents (eq.) of 1-ethyl-3-(3-dimethylaminopropyl)carbodiimide hydrochloride (EDC), 12 eq. of $N$-hydroxysuccinimide (NHS), and 10 eq. of ethylenediamine in nanopure water for $9 \mathrm{~h}$ with constant agitation using a rotor shaker (Scientific Industries, Bohemia, NY). Nanoparticles were washed twice by centrifugation $(10,000 \mathrm{rpm}$ for $5 \mathrm{~min}$ ) with the addition of nanopure water and sonication (at $40 \mathrm{~Hz}$ for $30 \mathrm{~s}$ ). The second reaction was performed at $4{ }^{\circ} \mathrm{C}$ in a nanoparticle suspension $(50 \mathrm{mg} / \mathrm{mL})$ in nanopure water with 12 eq. of EDC, 12 eq. of NHS and 10 eq. of the corresponding saccharide (i.e., galactose or di-mannose) or glycolic acid (linker between saccharide and nanoparticles; a control treatment for $\mathrm{AM} \varphi$ experiments) for $9 \mathrm{~h}$ with constant agitation. Nanoparticles were washed once and dried under vacuum for $6 \mathrm{~h}$. The automated set up was used to accurately dispense solutions of EDC, ethylenediamine, and NHS for the first reaction, and solutions of EDC, NHS, and saccharides in the second reaction to increase the throughput of the process. Particle morphology was characterized by scanning electron microscopy (SEM, FEI Quanta 250, Kyoto, Japan), and hydrodynamic size and $\zeta$-potential were determined by dynamic light scattering (DLS, Zetasizer Nano, Malvern Instruments Ltd., Worchester, UK). The saccharide concentration conjugated to the nanoparticles was measured using a high throughput phenol sulfuric acid assay as previously described. ${ }^{21,44}$

\subsection{Mice}

Wild type (WT) C57BL/6 (B6) mice were purchased from Harlan Laboratories (Indianapolis, IN) and macrophage mannose receptor deficient $\left(\mathrm{MMR}^{--}\right) \mathrm{B} 6$ mice were a generous gift from Dr. Mary Ann McDowell of the University of Notre Dame. Mice were housed in specific pathogen-free conditions where all bedding, caging, and feed were sterilized prior to use. All animal procedures were conducted with the approval of the Iowa State University Institutional Animal Care and Use Committee.

\subsection{Cell harvesting and culture}

Murine alveolar macrophages (AM $\varphi$ ) from WT and $\mathrm{MMR}^{-/-} \mathrm{B} 6$ mice were harvested by bronchoalveolar lavage as previously described. ${ }^{6,7,45}$ Briefly, mice were euthanized and a sterile catheter inserted into the trachea of each mouse. Using a $1 \mathrm{~mL}$ syringe fitted with the catheter, $0.75 \mathrm{~mL}$ of room temperature, sterile PBS was gently infused into the lungs and then aspirated back into syringe. This process was repeated six times while externally massaging the chest. Following collection, lavage fluid was immediately placed on ice prior to centrifugation $\left(250 \times \mathrm{g}, 10 \mathrm{~min}, 4^{\circ} \mathrm{C}\right)$. Cell viability was then assessed using trypan blue. Cells were cultured in Complete Tissue Culture Medium (CTCM) containing Dulbecco's Modified Eagle Medium with $4.5 \mathrm{mg}$ of glucose $/ \mathrm{mL}, 2 \mathrm{~mm}$ l-glutamine, $100 \mathrm{U}$ penicillin, $100 \mu \mathrm{g}$ streptomycin $/ \mathrm{mL}, 25 \mathrm{~mm}$ HEPES and $10 \%$ fetal bovine serum in six-well plates at a density of $5 \times 10^{5}$ cells/well for $8 \mathrm{~h}$ at $37{ }^{\circ} \mathrm{C}$ and $5 \% \mathrm{CO}_{2}$. After $6 \mathrm{~h}$ of incubation, non-adherent cells were discarded and adherent cells $(>90 \%)$ were incubated overnight prior to treatment Non-functionalized or functionalized nanoparticles were incubated with $\operatorname{AM} \varphi$ at a concentration of $0.125 \mathrm{mg} / \mathrm{mL}$. Non-stimulated $\mathrm{AM} \varphi$ and $\mathrm{AM} \varphi$ stimulated with Escherichia coli O111:B4 lipopolysaccharide (LPS, $200 \mathrm{ng} / \mathrm{mL}$ Sigma-Aldrich, St. Louis, MO) were used as negative and positive controls, respectively. After $48 \mathrm{~h}$, supernatants were collected for quantification of cytokines and nitrites. Cells were stained for flow cytometric analysis. Bone marrow-derived macrophages (BMM $\varphi$ ) were derived as previously described. ${ }^{46,47}$ Briefly, cells were obtained from WT and $\mathrm{MMR}^{--} \mathrm{B} 6$ mouse bone marrow and plated in a $150 \times 15 \mathrm{~mm}$ Petri dish with $30 \mathrm{~mL}$ CTCM supplemented with $30 \%$ L-cell conditioned medium. After 2 days, an additional $20 \mathrm{~mL}$ of CTCM with $30 \%$ L-cell conditioned medium was added to the cultures. At day 6 , adherent cell populations were harvested by placing the plates on ice for $20 \mathrm{~min}$ and then scraping with a cell scraper. After washing in PBS, live cells were counted and resuspended in CTCM prior to treatment with nanoparticles and stimulants as described for the AM $\varphi$.

\subsection{Cell surface marker evaluation}

Flow cytometric evaluation of cell surface markers was performed by modifying a previously described protocol. ${ }^{48}$ Briefly, cells were washed in $2 \mathrm{~mL}$ of fluorescence-activated cell sorting buffer (FACS buffer, $0.1 \%$ sodium azide and $0.1 \%$ bovine serum albumin in phosphate buffer saline). Fc $\gamma$ receptors were blocked with PBS containing $100 \mu \mathrm{g} / \mathrm{mL}$ rat anti-mouse CD16/CD32 antibody (BD Bioscience, San Diego, CA) and $1 \mathrm{mg} / \mathrm{mL}$ rat $\mathrm{IgG}$ for $30 \mathrm{~min}$ at $4{ }^{\circ} \mathrm{C}$ to prevent non-specific binding. $\operatorname{AM} \varphi$ were incubated with appropriate antibodies or isotype controls for $15 \mathrm{~min}$ on ice. Antibodies used for assessment of activation included phycoerythrin (PE)-Cy7 conjugated anti-mouse F4/80 (clone BM8), fluorescein isothiocyanate (FITC)-conjugated anti-mouse/ rat MHC II (I-A/I-E, clone M5/114.15.2), allophycocyanin (APC) anti-mouse CD40 (clone 1C10) and PE conjugated anti-mouse MHC I (H-2Kb, clone AF6-88.5.5.3). These antibodies and their respective isotype controls were purchased from eBioscience (San Diego, CA). APC-Cy7 anti-mouse CD86 (clone GL-1) was purchased from Biolegend (San Diego, CA). Antibodies used to evaluate CLR expression included F4/80, biotin conjugated anti-mouse 
CD209 (clone 5H10) and PE-Texas red conjugated streptavidin purchased from BD Biosciences, FITC anti-mouse CD206 (MMR, clone MR5D3) purchased from Biolegend and PE-CD301a/b (MGL1/2, affinity purified PAb catalog \# FAB4297P) purchased from R\&D systems (Minneapolis, MN). Samples were acquired using a FACSAria III flow cytometer (BD Biosciences) and the data were analyzed using FlowJo software (TreeStar Inc., Ashland, OR).

\subsection{Nanoparticle internalization}

At the time of fabrication, nanoparticles were loaded with cadmium selenide quantum dots (QDs; emission at $630 \mathrm{~nm}$ ) prior to functionalization. The QDs were kindly provided by Dr. Aaron Clapp of Iowa State University. QD-loaded functionalized and non-functionalized nanoparticles were used to stimulate $\operatorname{AM} \varphi$ for $48 \mathrm{~h}$. Cells were then analyzed by flow cytometry to identify the populations of $\mathrm{AM} \varphi$ that had phagocytosed nanoparticles (i.e., nanoparticlepositive versus nanoparticle-negative) as described previously. ${ }^{49}$ A QD control (background) was used to account for "false positives" because of QDs released due to nanoparticle degradation. QD-loaded, functionalized (e.g., dimannose) and non-functionalized nanoparticles were incubated in cell culture medium for $48 \mathrm{~h}$. After centrifugation $\left(250 \times \mathrm{g}, 10 \mathrm{~min}, 4{ }^{\circ} \mathrm{C}\right)$, supernatants were added to $\mathrm{AM} \varphi$. After $48 \mathrm{~h}$, the fluorescence registered for these control groups was considered as background. Cells were labeled with the above-described F4/80, MHC II, CD40, CD86 and CD301a/b antibodies as wells as with Pacific blue anti-mouse MHC I (H-2Kb, clone AF6-88.5) purchased from Biolegend, biotin anti-mouse CD209 and PerCP-Cy5.5 conjugated streptavidin purchased from eBioscience.

\subsection{Cytokine and reactive nitrogen species assays}

After stimulation for $48 \mathrm{~h}$ with non-functionalized or functionalized nanoparticles, cell-free supernatants were assayed for IL-1 $\beta$, IL-10, TNF- $\alpha$, IL-6, and IL-12p40 using a multiplex cytokine assay in conjunction with a Bio-Plex 200 System (Bio-Rad, Hercules, CA) as described elsewhere. ${ }^{33}$ Griess Reagent (Invitrogen, Carlsbad, CA) was used according to the manufacturer's specifications to determine nitrite concentration as an indirect method to measure production of reactive nitrogen species.

\subsection{Statistical analysis}

The statistical software $\mathrm{JMP}^{\circledR} 7$ was used to analyze all data. Tukey's HSD was used to determine significant differences among treatments. A student's $T$ test was employed to determine the significant differences between $\operatorname{AM} \varphi$ harvested from WT versus $\mathrm{MMR}^{-/-}$mice and between nanoparticle positive and negative AM $\varphi$. All $p$ values $<0.05$ were considered significant.

\section{Results}

\subsection{Synthesis and characterization of functionalized polyanhy- dride nanoparticles}

Amphiphilic 50:50 CPTEG:CPH copolymer was synthesized as described previously. ${ }^{31}$ The molecular weight $\left(M_{\mathrm{w}}\right)$ of the copolymer was $8000 \mathrm{Da}$ and ${ }^{1} \mathrm{H}$ NMR spectra of the copolymer were consistent with previously published data. ${ }^{31,33,35}$ Particle morphology was evaluated by SEM and was found to be consistent with previously published results (c.f. References [21], [33], \& [43]; and data not shown). The average diameter of the non-functionalized nanoparticles was $163 \pm 24 \mathrm{~nm}$ with a $\zeta$-potential of $-23 \pm 2.5 \mathrm{mV}$ (consistent with the presence of carboxylic acids on the surface). The glycolic acid (linker only) functionalized nanoparticles had an average diameter of $261 \pm 44 \mathrm{~nm}$ and a $\zeta$-potential of $20 \pm 2.7 \mathrm{mV}$, while di-mannose functionalized and galactose functionalized nanoparticles had average diameters of $271 \pm 40 \mathrm{~nm}$ and $263 \pm 44 \mathrm{~nm}$, respectively, with $\zeta$-potentials of $26 \pm 1.9 \mathrm{mV}$. The positive charges of the functionalized particles were consistent with previously published data $^{21}$ and are attributed to the presence of free ethylenediamine groups on the surface of the particles. Measuring the $\zeta$-potential of ethylenediamine-functionalized nanoparticles (determined to be $31 \pm 3.6 \mathrm{mV}$ ) further corroborated this result. These data indicate that partial capping of the ethylenediamine groups occurs when the saccharide is conjugated in the second reaction of the functionalization process. The di-mannose and galactose concentrations attached to the nanoparticles were normalized to the total mass of nanoparticles. The di-mannose concentration was $13.2 \pm 3.5 \mu \mathrm{g} / \mathrm{mg}$, consistent with previous work. ${ }^{21}$ The galactose concentration conjugated to the nanoparticles was $15.2 \pm 4.7 \mu \mathrm{g} / \mathrm{mg}$.

\subsection{Influence of functionalized polyanhydride nanoparticles on

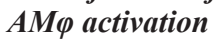

Previous work from our laboratories has highlighted the intrinsic adjuvant activity of polyanhydride micro and nanoparticles, as evidenced by their ability to activate DCs. ${ }^{48,}{ }^{49}$ In the current study, we observe, for the first time, increased expression of surface markers associated with antigen processing and presentation (MHC I and II) and T cell co-stimulation (CD86 and CD40) in primary $\mathrm{AM} \varphi$ cultured with non-functionalized (NF) polyanhydride nanoparticles as compared to non-stimulated AM $\varphi$ (Figure 1A-D). Culturing $\mathrm{AM} \varphi$ with non-functionalized polyanhydride nanoparticles also enhanced surface expression of CLRs (MMR, MGL and CIRE) in comparison to non-stimulated $\mathrm{AM} \varphi$ and $\mathrm{AM} \varphi$ stimulated with LPS (Figure 1E-G).

Functionalization of polyanhydride nanoparticles with either dimannose or galactose provided a significant enhancement in the expression of MHC I and CD40 on the surface of AM $\varphi$ in comparison to treatment with either non-functionalized particles or particles functionalized with only glycolic acid, the linker used to attach the carbohydrates to the nanoparticles (Figure 1A and D). AM $\varphi$ MHC II and CD86 expression was significantly increased over non-functionalized particles regardless of the nanoparticle functionalization (Figure $1 \mathrm{~B}$ and $\mathrm{C}$ ), with the greatest enhancement in MHC II observed on $\mathrm{AM} \varphi$ co-cultured with di-mannose functionalized particles (Figure 1B). As compared to non-functionalized nanoparticles, expression of the MMR on $\mathrm{AM} \varphi$ was only significantly enhanced upon incubation with di-mannose functionalized nanoparticles (Figure 1E). Similarly, co-culture with galactose functionalized nanoparticles significantly increased AM $\varphi$ MGL expression (Figure 1F). No significant enhancement in CIRE expression was observed when $\mathrm{AM} \varphi$ were incubated with functionalized versus non-functionalized nanoparticles (Figure 1G).

Together, these observations indicate that carbohydrate functionalization of polyanhydride nanoparticles enhanced $\mathrm{AM} \varphi$ activation as compared to non-functionalized particles. In some instances (e.g., MHC II and CD86 expression), functionalization with only the glycolic acid linker was sufficient to activate $A M \varphi$, indicating that nanoparticle surface charge contributes to $\operatorname{AM} \varphi$ activation. Finally, $\mathrm{AM} \varphi$ co-cultured with di-mannose or galactose functionalized particles exhibited enhanced cellular activation profiles that were comparable or superior to those observed in AM $\varphi$ stimulated with LPS.

\subsection{Relationship between functionalized polyanhydride nanoparti- cle internalization and CD40 expression on AM}

Phagocytosis of pathogenic bacteria is an important step associated with innate immune mechanisms and results in activated macrophages. ${ }^{50,51} \mathrm{We}$, therefore, evaluated the relationship between nanoparticle internalization and $\mathrm{AM} \varphi$ activation. ${ }^{49}$ Internalization of, and not just association with, nanoparticles by $\mathrm{AM} \varphi$ was confirmed by confocal microscopy (data not shown). Two populations of cells were identified-cells that internalized nanoparticles (QD-loaded nanoparticle-positive) and cells that did not internalize particles (nanoparticle-negative). Internalization of nanoparticles, regardless of functionalization status, was found to be required for the enhanced expression of $\mathrm{CD} 40$ on $\mathrm{AM} \varphi$ (Figure 2B), but not for the expression of CD86 (Figure 2A), MHC I, or MHC II (data not shown).

Interestingly, the nanoparticle-positive cells that internalized dimannose but not galactose-functionalized nanoparticles expressed significantly greater levels of the MMR as compared to nanoparticle-negative cells (Figure 2C). A similar relationship was observed for galactose but not di-mannose functionalized particles and MGL 

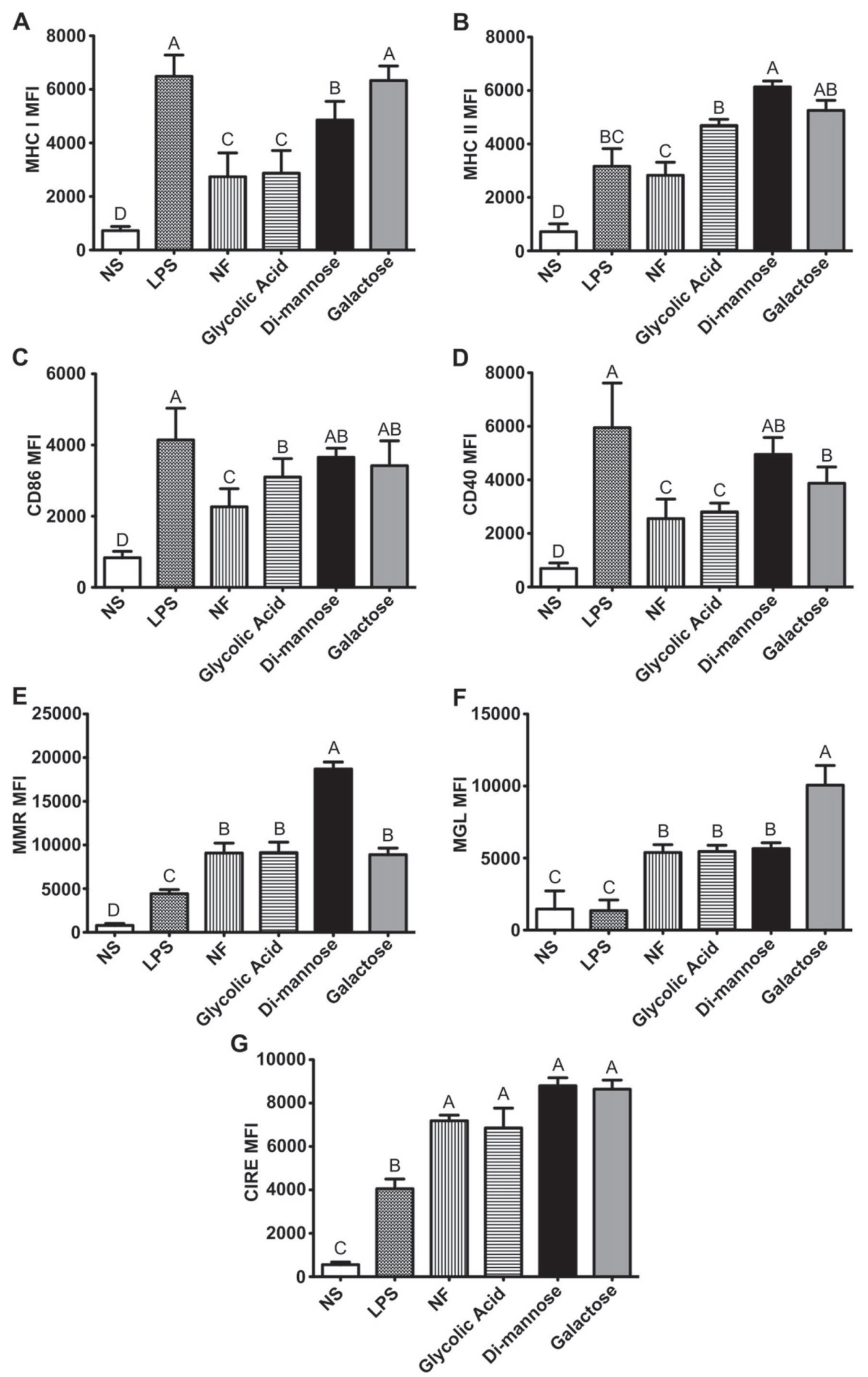

Figure 1. Functionalization of polyanhydride nanoparticles enhanced $\mathrm{AM} \varphi$ expression of MHC, T cell co-stimulatory molecules, and CLRs. After stimulation with non-functionalized (NF) or functionalized nanoparticles for $48 \mathrm{~h}$, AM $\varphi$ were harvested and analyzed by flow cytometry for surface expression of (A) MHC I, (B) MHC II, (C) CD86, (D) CD40, (E) MMR, (F) MGL, or (G) CIRE. LPS stimulated and non-stimulated cells (NS) were used as positive and negative controls, respectively. Data are expressed as the mean \pm the SEM of three independent experiments performed in triplicate. Treatments with different letters are significantly different from one another at $p<0.05 . \mathrm{MFI}=$ mean fluorescence intensity. 

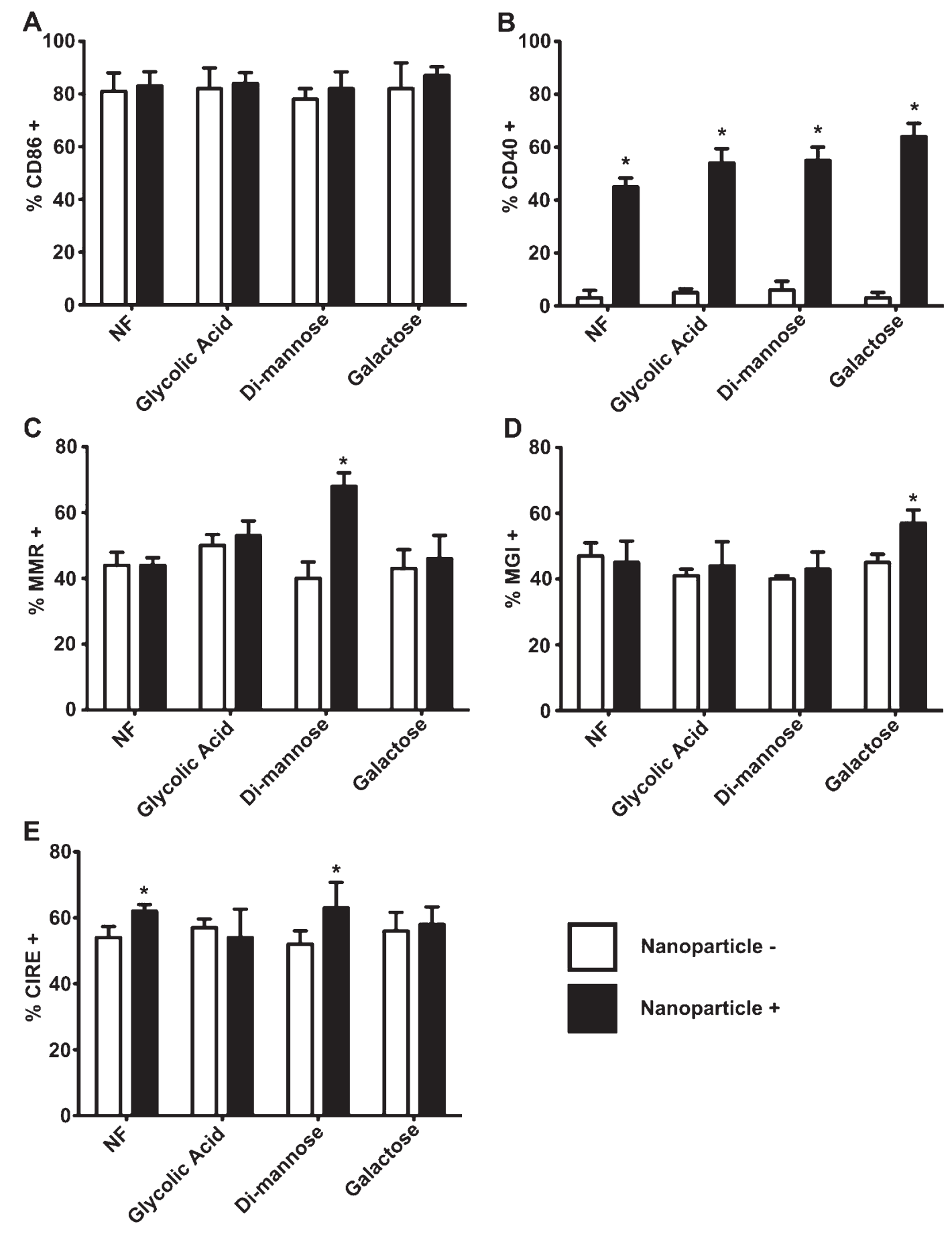

Figure 2. Internalization of functionalized polyanhydride nanoparticles was required for upregulation of CD40 expression on $\mathrm{AM} \varphi$. Percent of $\mathrm{AM} \varphi$ that did not internalize (nanoparticle-negative; 口) or did internalize (nanoparticle-positive; $\mathbf{m}$ ) nanoparticles after $48 \mathrm{~h}$ that were also positive for (A) CD86, (B) CD40, (C) MMR, (D) MGL, or (E) CIRE. Data are expressed as the mean \pm the SEM of three independent experiments performed in triplicate. * Represents a statistically significant difference between nanoparticle-positive and nanoparticle-negative populations within a treatment at $p<0.05$.

expression (Figure 2D). A significant increase in CIRE was observed on AM $\varphi$ that internalized either non-functionalized or dimannose functionalized nanoparticles but not nanoparticles that were functionalized with galactose or only the glycolic acid linker (Figure 2E). Together, these data support an association between internalization of nanoparticles functionalized with specific carbohydrates and the enhanced expression of the cognate receptor specific for that carbohydrate.

\subsection{Influence of functionalized polyanhydride nanoparticles on AM pro-inflammatory cytokine secretion}

Previous reports from our laboratory have described the ability of non-functionalized polyanhydride nanoparticles to enhance secretion of pro-inflammatory cytokines from APCs. ${ }^{32,}{ }^{48}$ In this present work, we sought to extend our findings by asking if functionalization 

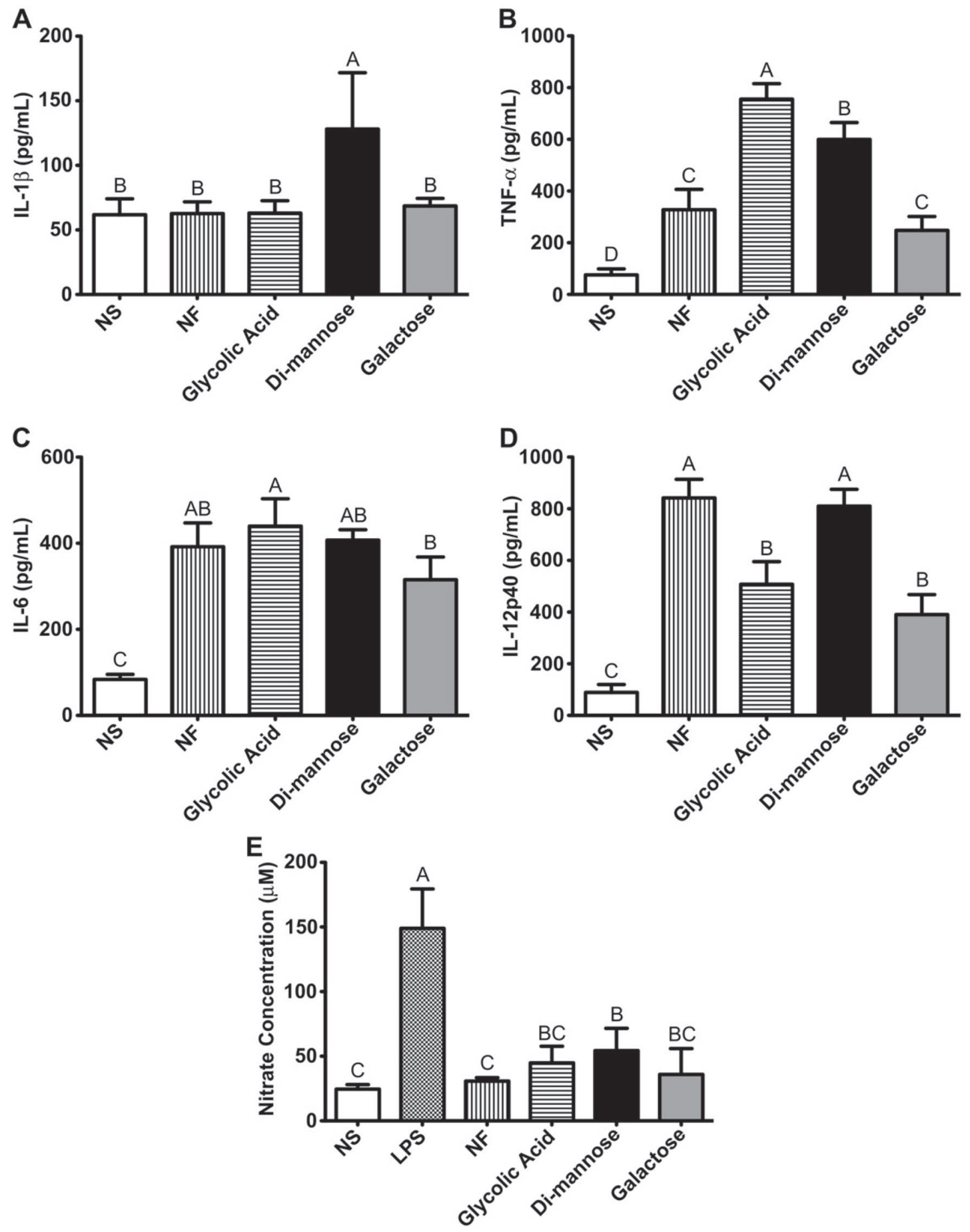

Figure 3. Carbohydrate functionalization of nanoparticles differentially influenced pro-inflammatory cytokine secretion. After stimulation with non-functionalized (NF) or functionalized nanoparticles for $48 \mathrm{~h}$, culture supernatants were harvested and assayed for (A) IL-1 $\beta$, (B) TNF- $\alpha$, (C) IL-6, or (D) IL-12p40. LPS stimulated and non-stimulated cells (NS) were used as positive and negative controls, respectively. Mean cytokine production for AM $\varphi$ stimulated with LPS: IL-1 $\beta=1021.7 \pm 66.5 \mathrm{pg} / \mathrm{mL}, \mathrm{IL}-6=7804.3 \pm 101.2 \mathrm{pg} / \mathrm{mL}, \mathrm{TNF}-\alpha=5711.5 \pm 181.7 \mathrm{pg} / \mathrm{mL}$, and IL-12p40 $=3637.8 \pm 111.6 \mathrm{pg} / \mathrm{mL}$. (E) Nitrate concentration was measured in culture supernatants via a Griess assay as an indirect method to quantify production of reactive nitrogen species. Data are expressed as the mean \pm the SEM of three independent experiments performed in triplicate. Treatments with different letters are significantly different from one another at $p<0.05$.

with specific carbohydrates provides additional stimulatory capacity to the polyanhydride nanoparticles. Indeed, di-mannose functionalized nanoparticles significantly elevated $\mathrm{AM} \varphi$ production of IL- $1 \beta$ as compared to all nanoparticle treatments (Figure 3A). Functionalization with either di-mannose or the glycolic acid linker but not galactose enhanced AM $\varphi$ secretion of TNF- $\alpha$ (Figure 3B). Di-mannose modification of the nanoparticles provided no additional benefit in terms of increasing IL-6 or IL-12p40 production (Figure 3C and D). Of note, functionalization with either the glycolic acid linker alone or galactose diminished the secretion of IL-12p40 observed when AM $\varphi$ were co-cultured with non-functionalized nanoparticles (Figure 3D). No IL-10 was detected in the culture supernatants of any AM $\varphi$ co- 
cultured with nanoparticles (data not shown). As shown in Figure 3E, the di-mannose functionalize particles induced significantly more reactive nitrogen species when compared to the non-stimulated group (negative control). All the other nanoparticle groups induced levels of reactive nitrogen species that were similar when compared to the non-stimulated group (negative control).

\subsection{Di-mannose functionalized nanoparticles and engagement of the MMR}

The data depicted in Figure 2 demonstrated that nanoparticle internalization was required for increased CD40 and CLR expression. Furthermore, we demonstrated that $\mathrm{AM} \varphi$ activation by functionalized nanoparticles was also a consequence of nanoparticle internalization (Figs. 1and 3). Using flow cytometry, we found that any functionalization of the nanoparticles enhanced uptake by $\operatorname{AM} \varphi$ as compared to non-functionalized particles (Figure 4, open bars).

To evaluate the role of the MMR on the uptake of di-mannose functionalized nanoparticles, we isolated $\mathrm{AM} \varphi$ from MMR-deficient $\left(\mathrm{MMR}^{-/}\right)$mice and co-cultured them with functionalized nanoparticles. As compared to wild type (WT) AM $\varphi$, a significant decrease in the number of internalized nanoparticles was only observed when di-mannose functionalized nanoparticles were co-cultured with $\mathrm{MMR}^{-1-} \mathrm{AM} \varphi$ (Figure 4, open versus closed bars). These data indicate that di-mannose functionalization of nanoparticles confers a specific interaction of the nanoparticles with the MMR that contributes to their enhanced internalization. In contrast, nanoparticles functionalized with galactose or only the glycolic acid linker are internalized via other, non-MMR-dependent pathways. Similar results were obtained using bone marrow-derived macrophages (BMM $\varphi$; Supplemental Figure 1).

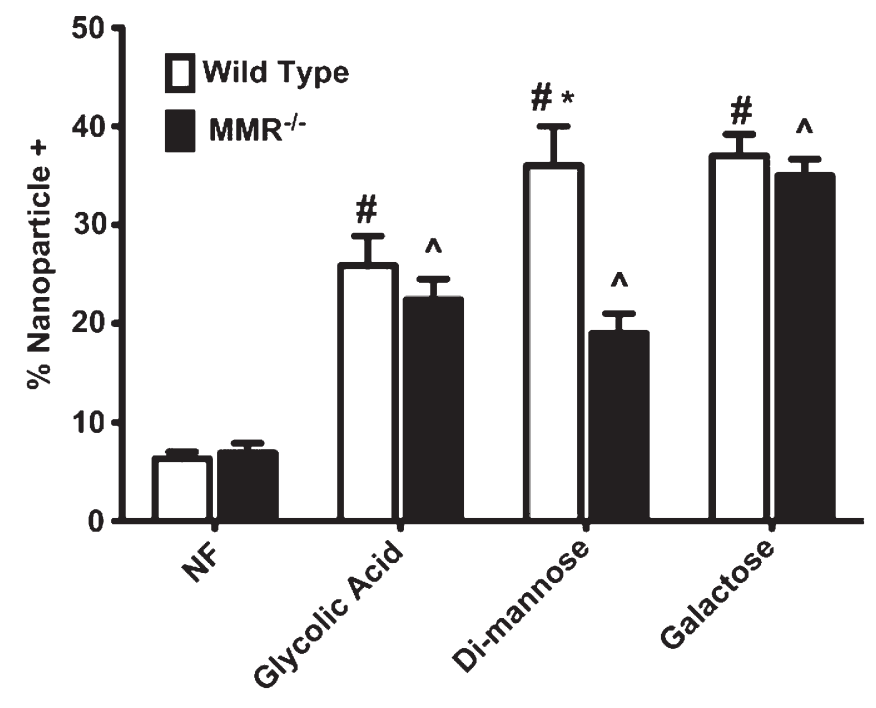

Figure 4. Di-mannose functionalized nanoparticles enhanced internalization by engaging the macrophage mannose receptor on $\operatorname{AM} \varphi$. Percent of wild

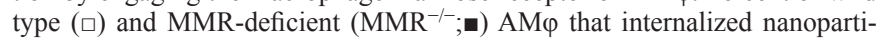
cles after $48 \mathrm{~h}$. Data are expressed as the mean \pm the SEM of three independent experiments performed in triplicate. * represents a statistically significant difference between wild type and $\mathrm{MMR}^{-/} \mathrm{AM} \varphi$ within a treatment at $p<0.05$. \# represents a statistically significant difference from the non-functionalized nanoparticle treatment group for wild type $\mathrm{AM} \varphi .^{\wedge}$ represents a statistically significant difference from the non-functionalized nanoparticle treatment group for $\mathrm{MMR}^{-/} \mathrm{AM} \varphi$.
Consistent with the internalization data, co-culture of WT AM $\varphi$ with di-mannose functionalized nanoparticles significantly increased the expression of MHC I, MHC II, CD86 and CD40 in comparison to that observed for $\mathrm{MMR}^{-1-} \mathrm{AM} \varphi$ (Figure 5A-D). Similarly, $\mathrm{MMR}^{-1-}$ AM $\varphi$ secreted significantly less IL-1 $\beta$ and IL- 6 as compared to WT $\operatorname{AM} \varphi$ when co-cultured with di-mannose functionalized nanoparticles (Figure 6A and $\mathrm{C}$ ). As compared to $\mathrm{WT} \mathrm{AM} \varphi$, reduced levels of CIRE expression (Figure 5E) and TNF- $\alpha$ and IL-12p40 secretion (Figure 6B and D) were also observed in $\mathrm{MMR}^{-1-} \mathrm{AM} \varphi$ co-cultured with di-mannose functionalized as well as with non-functionalized nanoparticles. In contrast, WT and $\mathrm{MMR}^{-/} \mathrm{AM} \varphi$ produced similar amounts of all cytokines measured following stimulation with LPS, indicating that $\mathrm{MMR}^{-/-} \mathrm{AM} \varphi$ were functionally capable of cytokine production (data not shown). Likewise, the absence of the MMR had no negative effect on the increased surface marker expression or cytokine production observed when $\mathrm{AM} \varphi$ were co-cultured with galactose functionalized nanoparticles (Figures $5 \& 6$ ), indicating that galactose functionalized particles do not require the MMR to promote $\operatorname{AM} \varphi$ activation. To rule out that our observations were unique to $\mathrm{AM} \varphi$, these analyses were performed using $\mathrm{BMM} \varphi$ and similar results were obtained (Supplemental Figures $2 \& 3$ ). Together, these data support the concept that di-mannose functionalized nanoparticles enhance $\mathrm{AM} \varphi$ activation by engaging the MMR.

\section{Discussion}

In the present work, we have designed an approach to targeted antigen delivery by functionalizing the surface of polyanhydride nanoparticles with specific carbohydrates to enable the nanoparticles to engage $\mathrm{C}$-type lectin receptors on $\mathrm{AM} \varphi$. Our rationale is that receptor-mediated engagement of nanoparticles will enhance their uptake and the activation of $\operatorname{AM} \varphi$, leading to the induction of robust immune responses in the respiratory tract.

Co-culture of functionalized nanoparticles with $\mathrm{AM} \varphi$ significantly increased cell surface expression of MHC I and II, CD86, CD40 and the C-type lectin receptor CIRE over non-functionalized nanoparticles (Figure 1A-D and G). Di-mannose and galactose functionalization also enhanced the expression of the MMR and MGL, respectively (Figure 1E and F). Carbohydrate modification also significantly increased uptake of the nanoparticles by $\mathrm{AM} \varphi$. Moreover, the enhanced expression of CD40 on AM $\varphi$ incubated with functionalized nanoparticles was found to be dependent upon nanoparticle internalization (Figure 2). The presence of nanoparticles in the coculture was sufficient for enhancement of certain activation markers (i.e., CD86, MHC I, and MHC II), while internalization was required for the expression of CD40. Our results show that the targeting of the C-type lectin receptors MMR, MGL, and CIRE resulted in a higher percentage of $\mathrm{AM} \varphi$ that internalize nanoparticles (Figure 4, open bars). This enhanced internalization may improve antigen delivery to antigen processing compartments ${ }^{52-54}$ and the enhancement of the expression of co-stimulatory receptors (i.e., CD86 and CD40) as shown in Figure 1.

Nanoparticle internalization also played a critical role in the enhanced expression of CLRs (Figure 2C-E). MMR expression was increased on AM $\varphi$ following co-culture with mannose-functionalized nanoparticles (Figure 1E), and this enhanced expression required internalization of the nanoparticles (Figure 2C). Similar results were observed for MGL (Figs. 1F and 2D). The expression of the CIRE receptor was enhanced by most of the nanoparticle formulations, but only cells that internalized non-functionalized and di-mannose functionalized nanoparticles showed increased expression of this marker (Figs. 1G and 2E). Several reports have shown that CLRs undergo internalization and recycling between the plasma membrane and the endosomal compartments. ${ }^{55-57}$ Our results indicate that positive selfregulation of specific CLRs occurs upon ligand recognition, subsequently increasing the expression of these markers. 

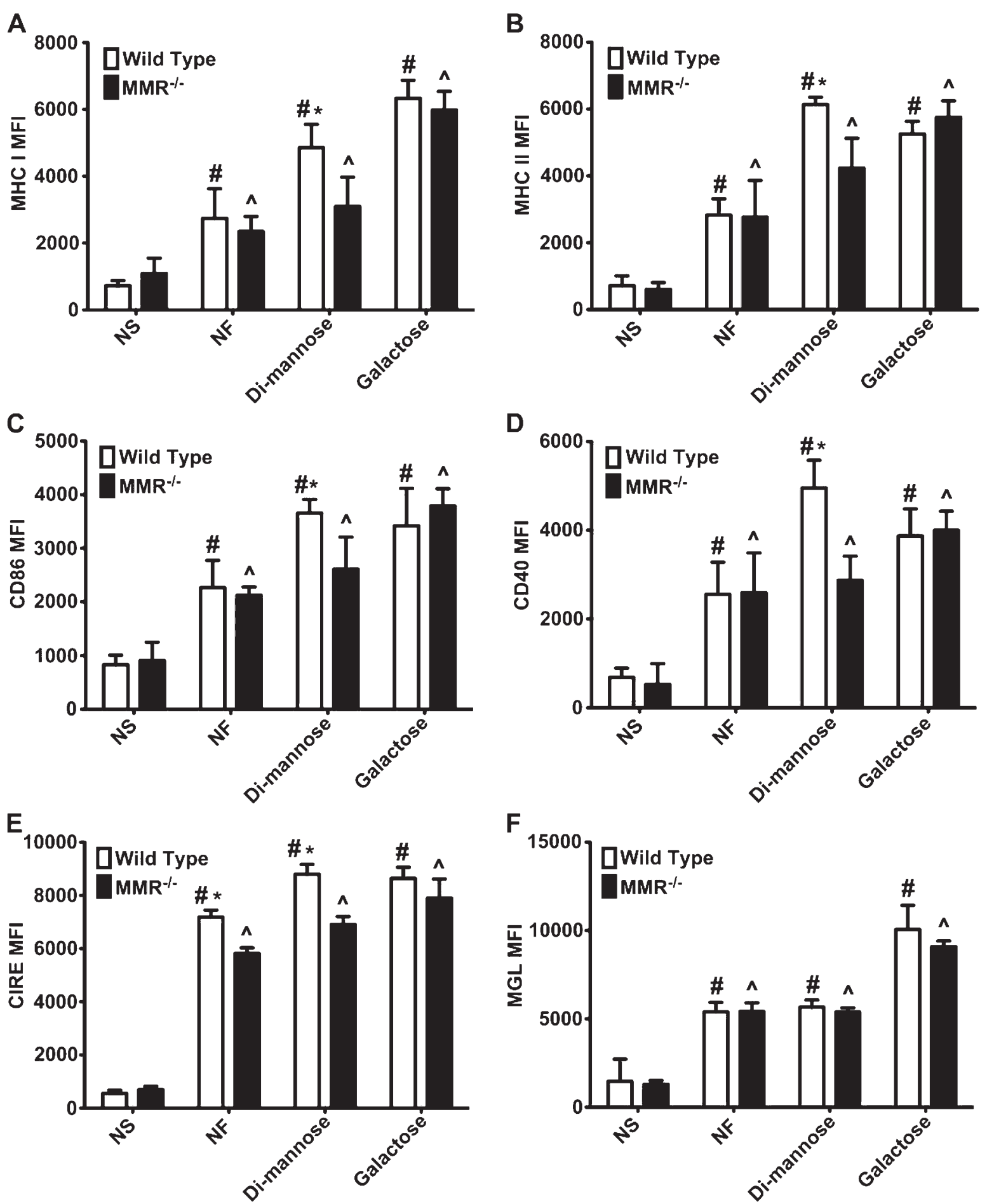

Figure 5. Di-mannose functionalized nanoparticles enhanced AM $\mathrm{A}$ expression of MHC, T cell co-stimulatory molecules and CLRs by engaging the macrophage mannose receptor. After stimulation with non-functionalized (NF) or functionalized nanoparticles for $48 \mathrm{~h}$, wild type ( $\square$ ) and MMR-deficient (MMR ${ }^{-/} ;-$) AM $\varphi$ were harvested and analyzed by flow cytometry for surface expression of (A) MHC I, (B) MHC II, (C) CD86, (D) CD40, (E) MGL, or (F) CIRE. Non-stimulated (NS) cells were used as negative controls. Data are expressed as the mean \pm the SEM of three independent experiments performed in triplicate. * Represents a statistically significant difference between wild type and $\mathrm{MMR}^{-/} \mathrm{AM} \varphi$ within a treatment at $p<0.05$. MFI $=$ mean fluorescence intensity. \# Represents a statistically significant difference from the NS cells for wild type AM $\varphi \cdot{ }^{\wedge}$ Represents a statistically significant difference from the NS cells for MMR ${ }^{-/-} \mathrm{AM} \varphi$.

The production of pro-inflammatory cytokines is essential for the activation of macrophages to stimulate their anti-microbial properties and for them to initiate adaptive immune responses. ${ }^{58}$ Di-mannose functionalization enhanced the production of IL-12p40, IL-1 $\beta$ and TNF- $\alpha$ (Figure 3A-D). These results are in agreement with studies demonstrating that the targeting of MMR resulted in the production of pro-inflammatory cytokines through the activation of NF- $\mathrm{BB} .{ }^{59}$ Although the production of pro-inflammatory cytokines was enhanced by the di-mannose functionalization, the levels secreted were modest enough to allay concerns about chronic inflammation in the 

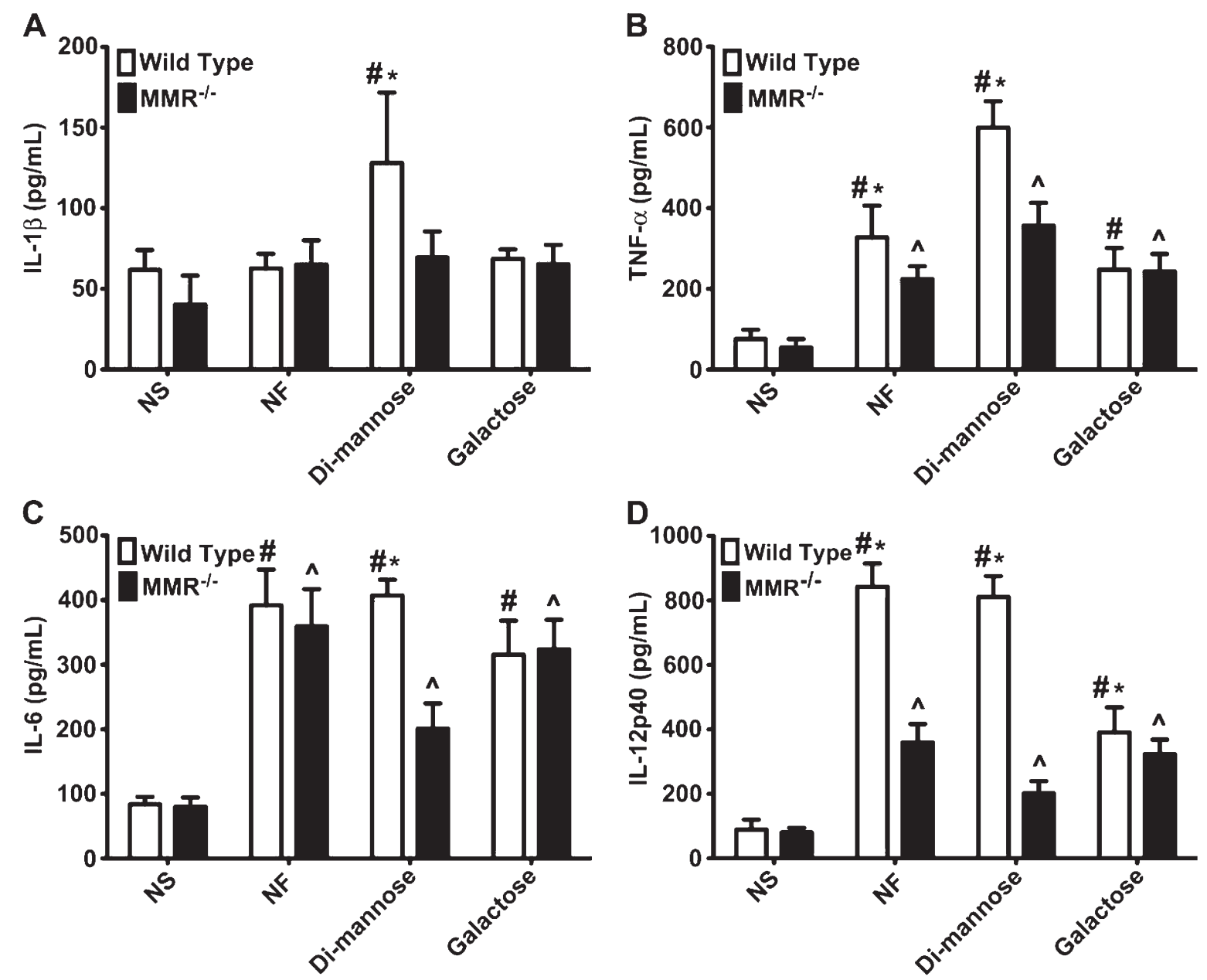

Figure 6. Di-mannose functionalized nanoparticles enhanced $\mathrm{AM} \varphi$ pro-inflammatory cytokine production by engaging the macrophage mannose receptor. After stimulation with non-functionalized (NF) or functionalized nanoparticles for $48 \mathrm{~h}$, culture supernatants from wild type ( $\square$ ) and MMR-deficient (MMR ${ }^{-/}$; $\boldsymbol{\text { - }}$ ) AM $\varphi$ were harvested and assayed for (A) IL-1 $\beta$, (B) TNF- $\alpha$, (C) IL-6, or (D) IL-12p40. Non-stimulated (NS) cells were used as a negative control. Data are expressed as the mean \pm the SEM of three independent experiments performed in triplicate. * Represents a statistically significant difference between wild type and $\mathrm{MMR}^{-/-} \mathrm{AM} \varphi$ within a treatment at $p<0.05$. \# Represents a statistically significant difference from the NS cells for wild type AM $\varphi .{ }^{\wedge} \operatorname{Represents}$ a statistically significant difference from the NS cells for $\mathrm{MMR}^{-/} \mathrm{AM} \varphi$.

respiratory tract following intranasal administration. The galactose functionalized nanoparticles resulted in an enhanced production of cytokines in comparison with the non-stimulated $\mathrm{AM} \varphi$, but at lower levels than the non-functionalized nanoparticles, particularly for IL$12 \mathrm{p} 40$. The release of pro-inflammatory cytokines when the cells were treated with galactose-functionalized particles was similar to that of the non-functionalized nanoparticles. These results are consistent with previous studies suggesting that galactose motifs enhanced the production of cytokines such as IL-12p40 and TNF- $\alpha$, but not to the levels obtained when mannose motifs are used. ${ }^{59,60}$

The elevated production of reactive nitrogen species has been related to chronic inflammation and cancers of several organs, including lungs. ${ }^{61}$ Such a response may be needed to combat an infection; however it would not be desirable consequence following administration of an intranasal vaccine. As shown in Figure 3E, all the nanoparticle groups induced levels of reactive nitrogen species that were similar when compared to the non-stimulated group (negative control). Collectively, these data demonstrate that di-mannose functionalization significantly enhanced the expression of cell surface makers and the production of relevant cytokines, leading to an activated phenotype of $A M \varphi$ that is necessary for the initiation of robust mucosal immune responses.
To further assess the role of MMR-specific recognition of dimannose functionalized nanoparticles, studies were performed using $\mathrm{AM} \varphi$ harvested from $\mathrm{MMR}^{-/}$mice. Statistically significant differences were observed in the internalization of di-mannose functionalized nanoparticles between $\mathrm{AM} \varphi$ harvested from wild type and $\mathrm{MMR}^{-/-}$mice (Figure 4), indicating that the MMR significantly contributes to the enhanced uptake observed when polyanhydride nanoparticles are functionalized with di-mannose. Enhanced uptake was also observed for glycolic acid functionalized nanoparticles as compared to non-functionalized nanoparticles. This may be attributed to the hydrophilic properties conferred by the mannose and glycolic acid groups to the surface of the particles that may increase their internalization in comparison to more hydrophobic surfaces (i.e., non-functionalized nanoparticles). This observation is consistent with previously published data that shows that hydrophilic chemistries are more readily internalized by $\mathrm{AM} \varphi$ and DCs. ${ }^{49,}{ }^{62}$ As expected, the absence of the MMR did not negatively affect the enhanced uptake of galactose-functionalized nanoparticles. The enhanced $\mathrm{AM} \varphi$ activation phenotype (i.e., above that induced by nonfunctionalized nanoparticles) observed following co-culture with di-mannose functionalized nanoparticles was also found to be dependent upon the presence of the MMR (Figures 5 \& 6). This finding 
suggests that specific engagement of the MMR further enhances the expression of cell surface markers and cytokine production. As indicated before, mannose is a component of the surface of many respiratory pathogens, including $Y$. pestis, $M$. tuberculosis, $S$. pneumoniae and influenza viruses, indicating that the MMR may be involved in how these pathogens are recognized by macrophages and DCs. Typically, upon encountering pathogens, these APCs upregulate expression of antigen presentation and co-stimulatory molecules as well as secrete cytokines to efficiently prime the naïve $T$ cells that help activate B cells. Our results indicate that di-mannose functionalized nanoparticles may have pathogen-like characteristics with respect to their APC activation abilities.

Together, these studies indicate that CLR targeting may be an effective strategy to activate $A M \varphi$ that, in turn, may be critical to the implementation of efficacious intranasal vaccines. Functionalized nanoparticles provide a versatile and robust platform that facilitates enhanced expression of antigen presentation and co-stimulatory molecules and secretion of the cytokines responsible for initiating and maintaining adaptive immunity. By innovatively engaging mechanisms or pathways that activate APCs, these studies indicate that it is feasible to develop an immunization regimen that safely and efficaciously induces protective immunity without the need to induce adverse or deleterious host responses.

\section{Conclusions}

The approach outlined in this present work demonstrates that rational design of efficacious vaccine adjuvants can be achieved by targeting CLRs on APCs. Specifically, we describe the functionalization of polyanhydride nanoparticles with two conserved carbohydrate structures commonly found on the surface of respiratory pathogens, di-mannose and galactose. The addition of these carbohydrates significantly enhanced the intrinsic adjuvant activity of our polyanhydride nanovaccine platform by further upregulating $\mathrm{AM} \varphi$ surface expression of MHC I and II, CLRs, the T cell co-stimulatory molecules CD86 and CD40 and the secretion of pro-inflammatory cytokines. Moreover, we demonstrate that the macrophage mannose receptor (MMR) played a central role in the activation of $\mathrm{AM} \varphi$ as well as the uptake of di-mannose functionalized polyanhydride nanoparticles. These studies provide important insights into the design, modification, and rational selection of intranasal vaccine carriers and/or adjuvants.

Acknowledgments-The authors would like to thank the United States Army Medical Research and Materiel Command for financial support (Grant No. W81XWH-10-1-0806). The authors are grateful to Shawn Rigby for his expertise in flow cytometry and to Dr. Mary Ann McDowell of the University of Notre Dame for generously providing the $\mathrm{MMR}^{-/}$mice. BN acknowledges the Balloun Professorship in Chemical and Biological Engineering and NLBP acknowledges the Wilkinson Professorship of Interdisciplinary Engineering.

\section{References}

[1] World Lung Foundation. ARIs overview, in The Acute Respiratory Infections Atlas, New York, NY, 2010.

[2] Ichinohe T, Ainai A, Nakamura T, Akiyama Y, Maeyama J, Odagiri T, et al. Induction of cross-protective immunity against influenza A virus $\mathrm{H} 5 \mathrm{~N} 1$ by an intranasal vaccine with extracts of mushroom mycelia. J Med Virol 2010; 82: 128-37.

[3] Lambkin R, Oxford JS, Bossuyt S, Mann A, Metcalfe IC, Herzog C, et al. Strong local and systemic protective immunity induced in the ferret model by an intranasal virosome-formulated influenza subunit vaccine. Vaccine $2004 ; 22: 4390-6$.

[4] Oliveira ML, Areas AP, Ho PL. Intranasal vaccines for protection against respiratory and systemic bacterial infections. Expert Rev Vaccines 2007; 6:419-29.

[5] Wimer-Mackin S, Hinchcliffe M, Petrie CR, Warwood SJ, Tino WT, Wil- liams MS, et al. An intranasal vaccine targeting both the Bacillus anthracis toxin and bacterium provides protection against aerosol spore challenge in rabbits. Vaccine 2006; 24:3953-63.

[6] Kirby AC, Coles MC, Kaye PM. Alveolar macrophages transport pathogens to lung draining lymph nodes. J Immunol 2009; 183:1983-9.

[7] Kirby AC, Raynes JG, Kaye PM. CD11b regulates recruitment of alveolar macrophages but not pulmonary dendritic cells after pneumococcal challenge. J Infect Dis 2006; 193:205-13.

[8] Worgall S, Leopold PL,Wolff G, Ferris B, Van Roijen N, Crystal RG. Role of alveolar macrophages in rapid elimination of adenovirus vectors administered to the epithelial surface of the respiratory tract. Hum Gene Ther 1997; 8:1675-84.

[9] Nicod LP. Pulmonary defence mechanisms. Respiration 1999; 66:2-11.

[10] Dobos KM, Swiderek K, Khoo KH, Brennan PJ, Belisle JT. Evidence for glycosylation sites on the 45-kilodalton glycoprotein of Mycobacterium tuberculosis. Infect Immun 1995; 63:2846-53.

[11] Hartley JL, Adams GA, Tornabene TG. Chemical and physical properties of lipopolysaccharide of Yersinia pestis. J Bacteriol 1974; 118:848-54.

[12] Lee CJ, Fraser BA, Szu S, Lin KT. Chemical structure of and immune response to polysaccharides of Streptococcus pneumoniae. Rev Infect Dis 1981; 3:323-31.

[13] Matsumoto A, Yoshima H, Kobata A. Carbohydrates of influenza virus hemagglutinin: structures of thewhole neutral sugar chains.Biochemistry $1983 ; 22: 188-96$.

[14] Schwarz RT, Schmidt MF, Anwer U, Klenk HD. Carbohydrates of influenza virus. I. Glycopeptides derived from viral glycoproteins after labeling with radioactive sugars. J Virol 1977; 23:217-26.

[15] Cambi A, Koopman M, Figdor CG. How C-type lectins detect pathogens. Cell Microbiol 2005; 7:481-8.

[16] Cambi A, Figdor CG. Dual function of C-type lectin-like receptors in the immune system. Curr Opin Cell Biol 2003; 15:539-46.

[17] Geijtenbeek TB, Gringhuis SI. Signalling through C-type lectin receptors: shaping immune responses. Nat Rev Immunol 2009; 9:465-79.

[18] Lee MS, Kim YJ. Signaling pathways downstream of pattern-recognition receptors and their cross talk. Annu Rev Biochem 2007; 76:447-80.

[19] McGreal EP, Miller JL, Gordon S. Ligand recognition by antigen-presenting cell C-type lectin receptors. Curr Opin Immunol 2005; 17:18-24.

[20] van Kooyk Y, Rabinovich GA. Protein-glycan interactions in the control of innate and adaptive immune responses. Nat Immunol 2008; 9:593-601.

[21] Carrillo-Conde B, Song E, Chavez-Santoscoy A, Phanse Y, Ramer-Tait A, Pohl N, et al. Mannose-funtionalized "pathogen-like" polyanhydride nanoparticles target C-type lectin receptors on dendritic cells. Mol Pharm 2011; 8:1877-86.

[22] Cruz LJ, Tacken PJ, Bonetto F, Buschow SI, Croes HJ, Wijers M, et al. Multimodal imaging of nanovaccine carriers targeted to human dendritic cells. Mol Pharma 2011; 8:520-31.

[23] Irache JM, Salman HH, Gamazo C, Espuelas S. Mannose-targeted systems for the delivery of therapeutics. Expert Opin Drug Deliv 2008; 5:703-24.

[24] Jiang HL, Kang ML, Quan JS, Kang SG, Akaike T, Cho CS, et al. The potential of mannosylated chitosan microspheres to target macrophage mannose receptors in an adjuvant-delivery system for intranasal immunization. Biomaterials 2008; 29:1931-9.

[25] Keler T, Ramakrishna V, Fanger MW. Mannose receptor-targeted vaccines. Expert Opin Biol Ther 2004; 4:1953-62.

[26] Pietrella D, Corbucci C, Perito S, Bistoni G, Vecchiarelli A. Mannoproteins from Cryptococcus neoformans promote dendritic cell maturation and activation. Infect Immun 2005; 73:820-7.

[27] Salman HH, Irache JM, Gamazo C. Immunoadjuvant capacity of flagellin and mannosamine-coated poly(anhydride) nanoparticles in oral vaccination. Vaccine 2009; 27:4784-90.

[28] Singh SK, Stephani J, Schaefer M, Kalay H, Garcia-Vallejo JJ, den Haan $\mathrm{J}$, et al. Targeting glycan modified OVA to murine DC-SIGN transgenic dendritic cells enhances MHC class I and II presentation. Mol Immunol $2009 ; 47: 164-74$.

[29] Tang CK, Sheng KC, Apostolopoulos V, Pietersz GA. Protein/peptide and DNA vaccine delivery by targeting C-type lectin receptors. Expert Rev Vaccines 2008; 7:1005-18. 
[30] Conix A. Poly[1,3-bis(p-carxoyphenoxy)-propane anhydride]. Macro Synth; 1966:95-8.

[31] Torres M, Vogel B, Narasimhan B, Mallapragada S. Synthesis and characterization of novel polyanhydrides with tailored erosion mechanism. J Biomed Mater Res A 2005; 76A:102-10.

[32] Petersen LK, Sackett CK, Narasimhan B. A novel high-throughput screening method to study the effect of device geometry, polymer chemistry and $\mathrm{pH}$ on in vitro protein release from polyanhydrides. J Comb Chem 2010; 12:51-6.

[33] Petersen LK, Xue L, Wannemuehler MJ, Rajan K, Narasimhan B. The simultaneous effect of polymer chemistry and device geometry on the in vitro activation of murine dendritic cells. Biomaterials 2009; 30:5131-42.

[34] Thorstenson JB, Petersen LK, Narasimhan B. Combinatorial/high throughput methods for the determination of polyanhydride phase behavior. J Comb Chem 2009; 11:820-8.

[35] Vogel BM, Cabral JT, Eidelman N, Narasimhan B, Mallapragada SK. Parallel synthesis and high throughput dissolution testing of biodegradable polyanhydride copolymers. J Comb Chem 2005; 7:921-8.

[36] Jaipuri FA, Pohl NL. Toward solution-phase automated iterative synthesis: fluorous-tag assisted solution-phase synthesis of linear and branched mannose oligomers. Org Biomol Chem 2008; 6:2686-91.

[37] Schmidt RR, Jung K. Trichloroacetimidates: chemistry of saccharides. carbohydrates in chemistry and biology. Weinheim, Germany: WileyVCH Verlag GmbH; 2000.

[38] Song EH, Osanya AO, Petersen CA, Pohl NL. Synthesis of multivalent tuberculosis and Leishmania-associated capping carbohydrates reveals structuredependent responses allowing immune evasion. J Am Chem Soc 2010; 132: 11428-30.

[39] Park WKC, Auer M, Jaksche H, Wong CH. Rapid combinatorial synthesis of aminoglycoside antibiotic mimetics: use of a polyethylene glycol-linked amine and a neamine-derived aldehyde in multiple component condensation as a strategy for the discovery of new inhibitors of the HIV RNA Rev responsive element. J Am Chem Soc; 1996:10150-5.

[40] Sheehan J, Cruickshank P, Boshart G. Notes-A convenient synthesis of watersoluble carbodiimides. J Org Chem 1961; 26:2525-8.

[41] Carlsen HJ, Katsuki T, Martin VS, Sharpless B. A greatly improved procedure for ruthenium tetroxide catalyzed oxidations of organic compounds. J Org Chem 1981; 46:3936-8.

[42] Ghosh M, Dulina RG, Kakarla R, Sofia MJ. Efficient synthesis of a stereochemically defined carbohydrate scaffold: carboxymethyl 2-acetamido-6- azido-4-O-benzyl-2-deoxy-alpha-D-glucopyranoside. J Org Chem 2000; 65: 8387-90.

[43] Ulery BD, Phanse Y, Sinha A, Wannemuehler MJ, Narasimhan B, Bellaire BH. Polymer chemistry influences monocytic uptake of polyanhydride nanospheres. Pharm Res 2009; 26:683-90.

[44] Masuko T, Minami A, Iwasaki N, Majima T, Nishimura S, Lee YC. Carbohydrate analysis by a phenol-sulfuric acid method in microplate format. Anal Biochem 2005; 339:69-72.

[45] Suzuki M, Tachibana I, Takeda Y, He P, Minami S, Iwasaki T, et al. Tetraspanin CD9 negatively regulates lipopolysaccharide-induced macro- phage activation and lung inflammation. J Immunol 2009; 182:6485-93.

[46] Mukbel R, Petersen CA, Jones DE. Soluble factors from Leishmania majorspecific CD4p T cells and B cells limit L. amazonensis amastigote survival within infected macrophages. Microbes Infect 2006; 8:2547-55.

[47] Racoosin EL, Swanson JA. Macrophage colony-stimulating factor (rMCSF) stimulates pinocytosis in bone marrow-derived macrophages. J Exp Med 1989; 170:1635-48.

[48] Torres M, Wilson-Welder J, Lopac SK, Phanse Y, Carrillo-Conde B, Ramer- Tait A, et al. Polyanhydride microparticles enhance dendritic cell antigen presentation and activation. Acta Biomaterialia 2011; 7:2857-64.

[49] Petersen LK, Ramer-Tait AE, Broderick SR, Kong CS, Ulery BD, Rajan $\mathrm{K}$, et al. Activation of innate immune responses in a pathogen-mimicking manner by amphiphilic polyanhydride nanoparticle adjuvants. Biomaterials 2011; 32: 6815-22.

[50] Aderem A, Underhill DM. Mechanisms of phagocytosis in macrophages. Annu Rev Immunol 1999; 17:593-623.

[51] Schnyder J, Baggiolini M. Role of phagocytosis in the activation of macrophages. J Exp Med 1978; 148:1449-57.

[52] Cambi A, Beeren I, Joosten B, Fransen JA, Figdor CG. The C-type lectin DC-SIGN internalizes soluble antigens and HIV-1 virions via a clathrindependent mechanism. Eur J Immunol 2009; 39:1923-8.

[53] Lanzavecchia A. Mechanisms of antigen uptake for presentation. Curr Opin Immunol 1996; 8:348-54.

[54] van Vliet SJ, Saeland E, van Kooyk Y. Sweet preferences of MGL: carbohydrate specificity and function. Trends Immunol 2008; 29:83-90.

[55] Geijtenbeek TB, van Vliet SJ, Engering A, 't Hart BA, van Kooyk Y. Self- and nonself-recognition by C-type lectins on dendritic cells. Annu Rev Immunol 2004; 22:33-54.

[56] Pyz E, Marshall AS, Gordon S, Brown GD. C-type lectin-like receptors on myeloid cells. Ann Med 2006; 38:242-51.

[57] Robinson MJ, Sancho D, Slack EC, LeibundGut-Landmann S, Reis e Sousa C. Myeloid C-type lectins in innate immunity. Nat Immunol 2006; 7:1258-65.

[58] Cavaillon JM. Cytokines and macrophages. Biomed Pharmacother 1994; 48: $445-53$.

[59] Zhang J, Zhu J, Imrich A, Cushion M, Kinane TB, Koziel H. Pneumocystis activates human alveolar macrophage NF-kappaB signaling through mannose receptors. Infect Immun 2004; 72:3147-60.

[60] Kean DE, Ohtsuka I, Sato K, Hada N, Takeda T, Lochnit G, et al. Dissecting Ascaris glycosphingolipids for immunomodulatory moieties-the use of synthetic structural glycosphingolipid analogues. Parasite Immunol 2006; 28: 69-76.

[61] Dedon PC, Tannenbaum SR. Reactive nitrogen species in the chemical biology of inflammation. Arch Biochem Biophys 2004; 423:12-22.

[62] Walter E, Dreher D, Kok M, Thiele L, Kiama SG, Gehr P, et al. Hydrophilic poly(DL-lactide-co-glycolide) microspheres for the delivery of DNA to human-derived macrophages and dendritic cells. J Control Release 2001; 76:149-68.

Appendix A, containing Supplemental Figures 1-3, follows. 


\section{Supplemental Figure 1}

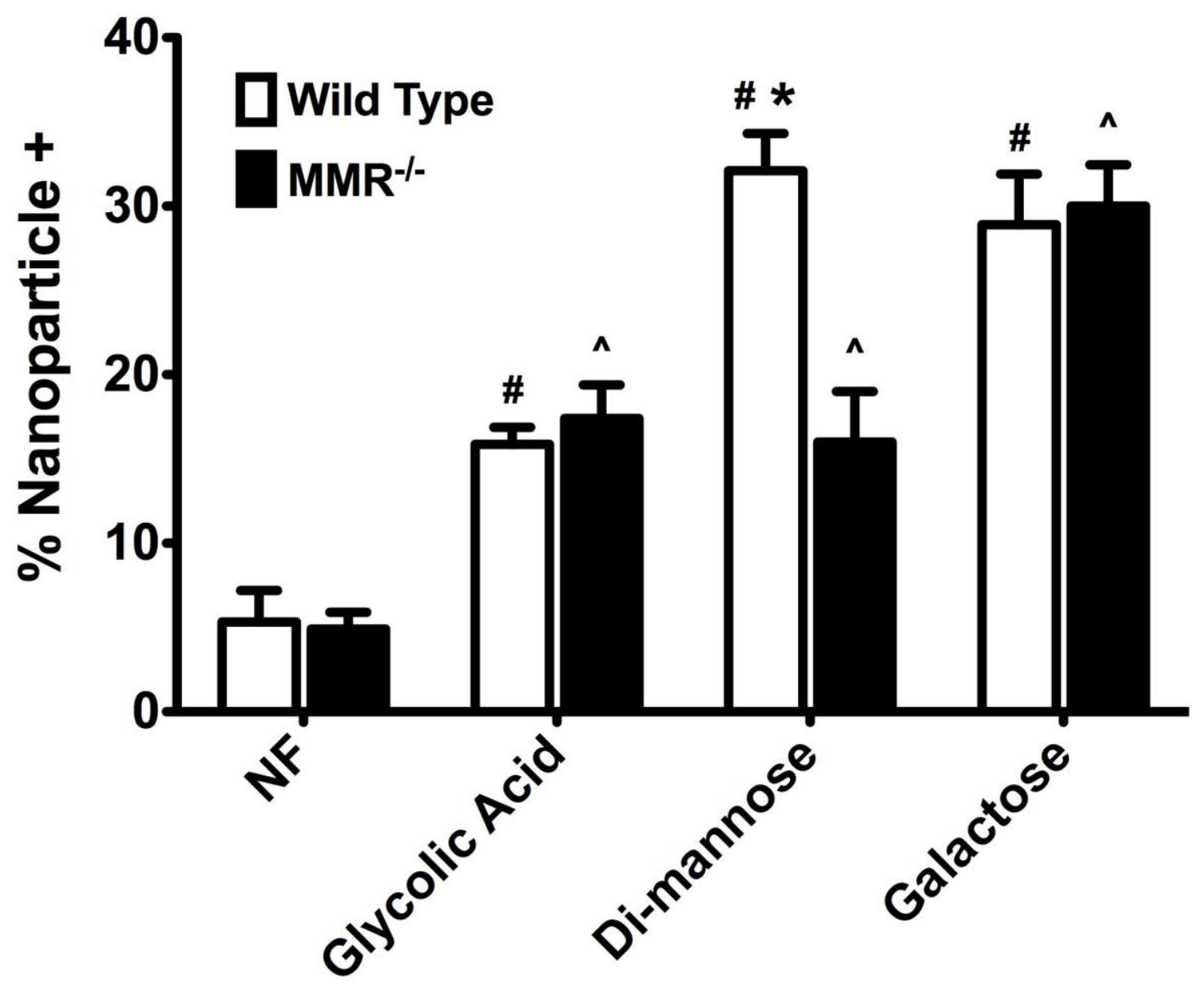

Supplemental Figure 1. Di-mannose functionalized nanoparticles enhanced internalization by engaging the macrophage mannose receptor on bone marrow-derived macrophages (BMMф). Percent of wild type ( $\square$ ) and MMR-deficient $\left(\mathrm{MMR}^{--} ; \mathbf{\square}\right)$ BMM $\phi$ that internalized nanoparticles after $48 \mathrm{~h}$. Data are expressed as the mean \pm the SEM of three independent experiments performed in triplicate. ${ }^{*}$ represents a statistically significant difference between wild type and $\mathrm{MMR}^{-/-} \mathrm{BMM} \phi$ within a treatment at $\mathrm{p}<0.05$. \# represents a statistically significant 
difference from the non-functionalized nanoparticle treatment group for wild type BMM $\phi .{ }^{\wedge}$ represents a statistically significant difference from the non-functionalized nanoparticle treatment group for $\mathrm{MMR}^{-1-} \mathrm{AM} \phi$. 


\section{Supplemental Figure 2}

A

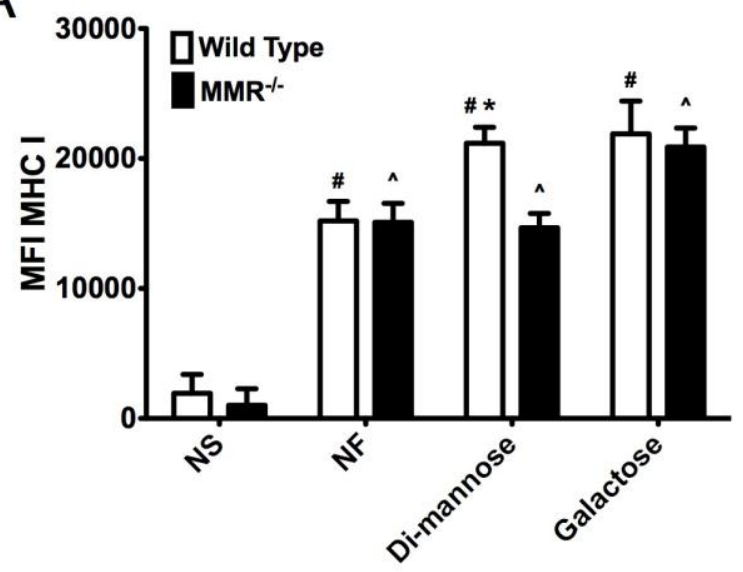

C

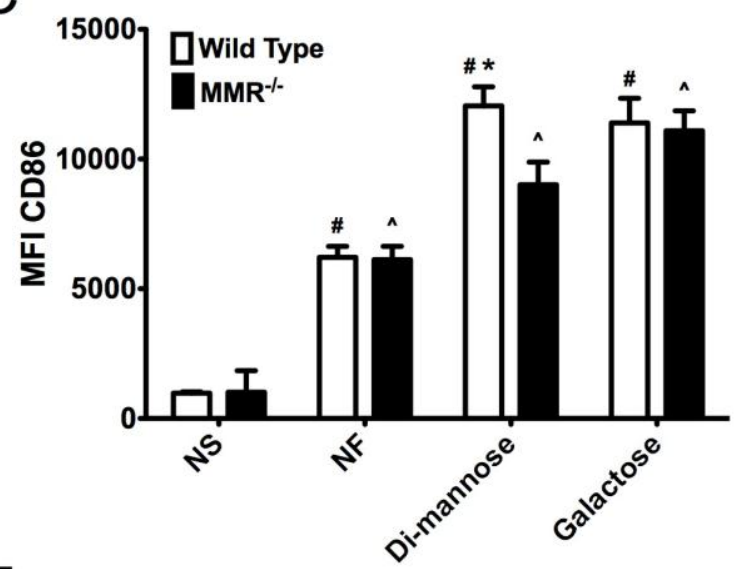

E

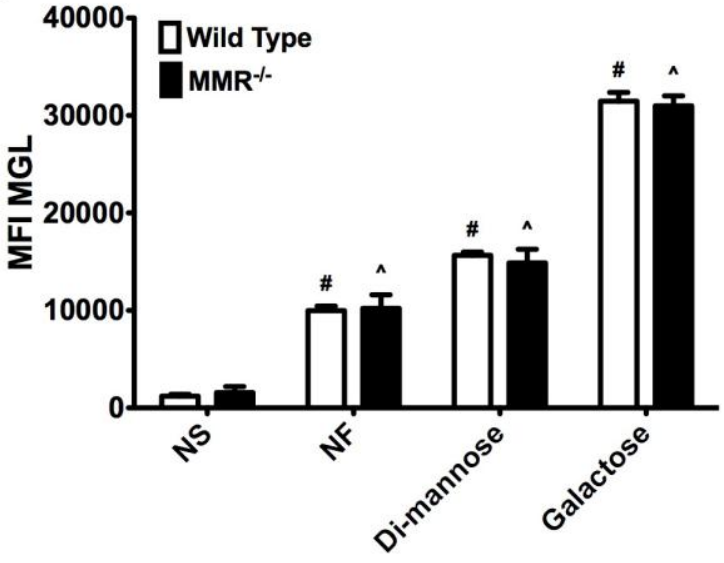

B

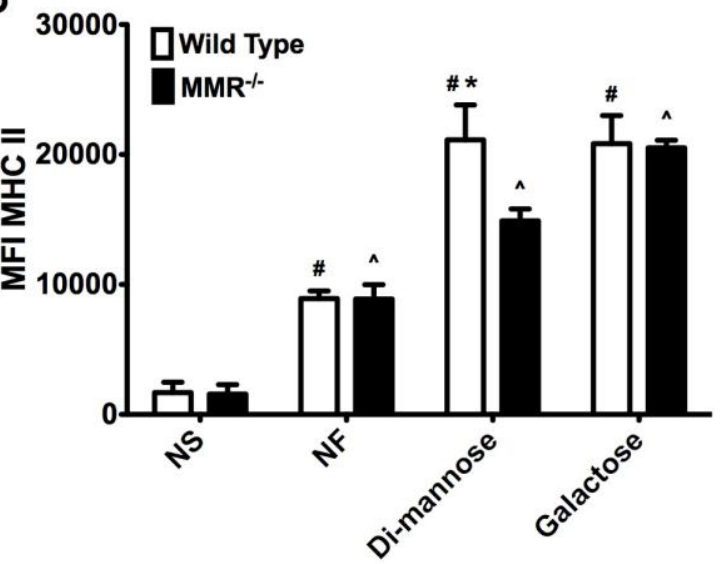

D

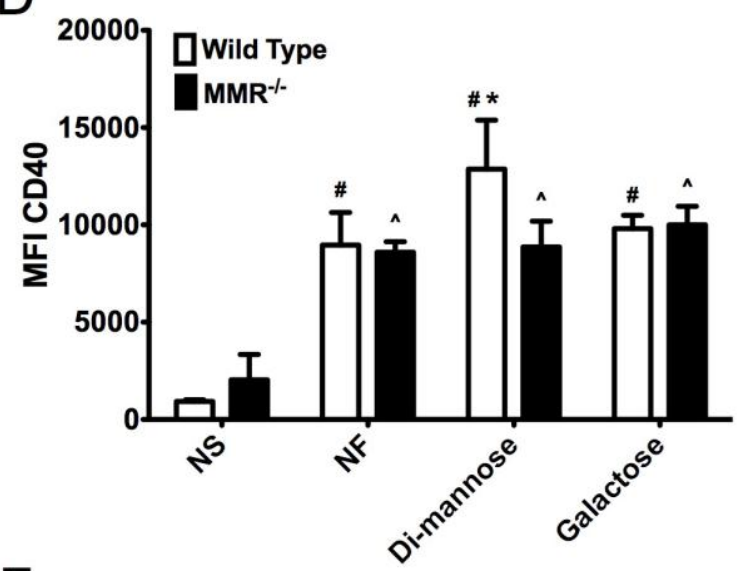

$\mathrm{F}$

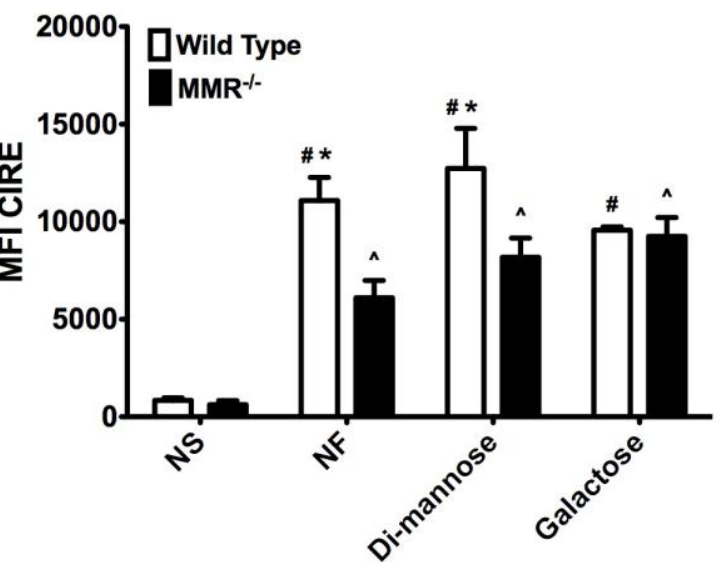


Supplemental Figure 2. Di-mannose functionalized nanoparticles enhanced bone marrowderived macrophage (BMM $\Phi)$ expression of MHC, T cell co-stimulatory molecules and CLRs by engaging the macrophage mannose receptor. After stimulation with nonfunctionalized (NF) or functionalized nanoparticles for $48 \mathrm{~h}$, wild type $(\square)$ and MMR-deficient $\left(\mathrm{MMR}^{--} ; \mathbf{\square}\right) \mathrm{BMM} \phi$ were harvested and analyzed by flow cytometry for surface expression of $(\mathrm{A})$ MHC I, (B) MHC II, (C) CD86, (D) CD40, (E) MGL, or (F) CIRE. Non-stimulated (NS) cells were used as negative controls. Data are expressed as the mean \pm the SEM of three independent experiments performed in triplicate. * represents a statistically significant difference between wild type and $\mathrm{MMR}^{-/} \mathrm{BMM} \phi$ within a treatment at $\mathrm{p}<0.05 . \mathrm{MFI}=$ mean fluorescence intensity. \# represents a statistically significant difference from the NS cells for wild type AM $\phi .{ }^{\wedge}$ represents a statistically significant difference from the NS cells for $\mathrm{MMR}^{-/} \mathrm{AM} \phi$. 


\section{Supplemental Figure 3}
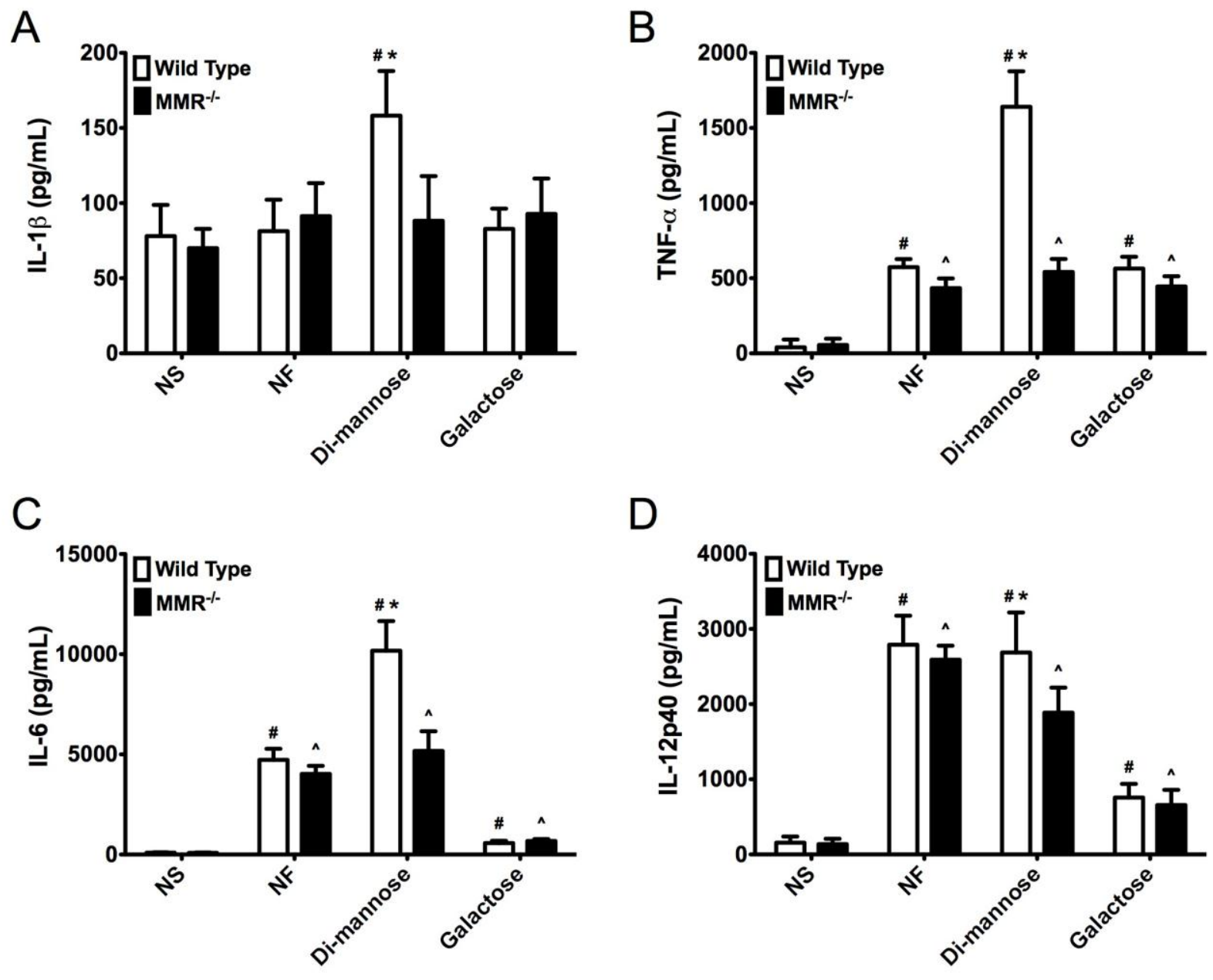

Supplemental Figure 3. Di-mannose functionalized nanoparticles enhanced bone marrowderived macrophage (BMM $\Phi$ ) pro-inflammatory cytokine production by engaging the macrophage mannose receptor. After stimulation with non-functionalized (NF) or functionalized nanoparticles for $48 \mathrm{~h}$, culture supernatants from wild type ( $\square$ ) and MMR-deficient $\left(\mathrm{MMR}^{-/-} ; \boldsymbol{\square}\right)$ BMMф were harvested and assayed for $(\mathrm{A}) \mathrm{IL}-1 \beta,(\mathrm{B})$ TNF- $\alpha,(\mathrm{C}) \mathrm{IL}-6$, or (D) IL12p40. Non-stimulated (NS) cells were used as a negative control. Data are expressed as the mean \pm the SEM of three independent experiments performed in triplicate. * represents a statistically significant difference between wild type and $\mathrm{MMR}^{-/-}$BMM $\phi$ within a treatment at $\mathrm{p}<$ 
0.05. \# represents a statistically significant difference from the NS cells for wild type AM $\phi . \wedge$ represents a statistically significant difference from the NS cells for $\mathrm{MMR}^{-/-} \mathrm{AM} \phi$. 